\title{
Multi-Functional Measurement Using a Single FBG Sensor
}

\author{
Y. Mizutani • R.M. Groves
}

Received: 5 July 2010 /Accepted: 13 January 2011 / Published online: 8 March 2011

(C) The Author(s) 2011. This article is published with open access at Springerlink.com

\begin{abstract}
This paper describes the measurement of average strain, strain distribution and vibration of a cantilever beam made of Carbon Fiber Reinforced Plastics (CFRP), using a single Fibre Bragg Grating (FBG) sensor mounted on the beam surface. Average strain is determined from the displacement of the peak wavelength of reflected spectrum from the FBG sensor. Two unstrained reference FBG sensors were used to compensate for temperature drift. Measured strains agree with those measured by a resistance foil strain gauge attached to the sample. Stress distributions are measured by monitoring the variation in the full width at half maximum (FWHM) values of the reflected spectrum, using a proposed optical analytical model, described in the paper. FWHM values were measured for both the cantilever test beam and for a reference beam, loaded using a four-point bending rig. The trend of the stress distribution for the test beam matches with our analytical model, however with a relatively large noise present in the
\end{abstract}

\section{Y. Mizutani}

Faculty of Aerospace Engineering,

Delft University of Technology,

Kluyverweg 1,

2600 GB Delft, The Netherlands

Y. Mizutani $(\bowtie)$

Department of Mechanical Sciences and Engineering,

Tokyo Institute of Technology,

2-12-1-I1-70, Ookayama, Meguro-ku,

Tokyo 152-855, Japan

e-mail: ymizutan@mes.titech.ac.jp

\section{R.M. Groves}

Optical Non-Destructive Testing Laboratory,

Faculty of Aerospace Engineering,

Delft University of Technology,

Kluyverweg 1,

2600 GB Delft, The Netherlands experimentally determined data. The vibration of a cantilever beam was measured by temporal analysis of the peak reflection wavelength. This technique is very stable as measurements are not affected by variations in the signal amplitude. Finally an application of FBG sensors for damage detection of CFRP plates, by measuring the natural frequency, is demonstrated. With small defects of different sizes applied to the CFRP plate, the natural frequency decreased with damage size.

Keywords Fibre Bragg grating · Carbon fiber reinforced plastic $\cdot$ Strain measurement $\cdot$ Strain distribution $\cdot$ Natural frequency Damage detection

\section{Introduction}

In recent years, Fibre Bragg Grating (FBG) sensors $[1,2]$ have been used for static and dynamic strain measurements of industrial structures due to their characteristics of lightness, inertness to electric or magnetic inference and multiplexing capability [3, 4].

Monitoring of strain by an FBG sensor is conducted by measuring the shift in Bragg wavelength of the sensor. Since the Bragg wavelength is changed by both strain and temperature variations, temperature compensation is required for accurate strain measurements. Several methods have been proposed to compensate for this temperature effect [5-7]. Xu et al. [5] prepared special superimposed fibre gratings having different Bragg wavelengths to measure strain and temperature separately. By monitoring the shift in Bragg wavelength of two different FBGs experiencing the same strain and temperature conditions, and using the difference of the strain-optic and thermo-optic coefficients, they successfully measured strain and temperature 
separately. Chiang et al. [6] used two FBG sensors with identical, or similar, Bragg wavelengths as a sensor, putting them under the same temperature conditions but with one FBG kept free from strain. They measured the change of the reflected spectrum intensity and estimated strains without the temperature effect. Takahashi et al. [7] used two FBG sensors, one for sensing and the other for filtering the optical source, in combination with a broad-spectrum light source and optical circulator to compensate for the temperature effect. Dias et al. [8] used an electronic technique for temperature compensation. They measured the shift of the Bragg wavelength in a reference FBG due to temperature with an electronic feedback control circuit and controlled the wavelength of a laser diode by changing the laser temperature to compensate for the effect. These techniques are sufficient to measure the strain without the temperature effect, although a much more simple methodology and system are desired.

In general, the shift of the Bragg wavelength in an FBG is measured by a scanning wavelength source, static or scanning optical filters or interferometry [3]. The scanning source and filter methods are stable and accurate for static strain measurement. The optical filtering method and interferometric methods are more adapted for dynamic strain measurement. However, due to recent technology improvements of measurement instruments, such as the increase in memory of digitizers and computers, there is a possibility to measure dynamics strain also by the scanning filter method.

Several researches are focused on strain distribution measurement by using changes of the shape of the reflection spectrum. In several papers [9-12], reflected spectral shapes with non-uniform strain distribution on FBG fiber are calculated using intensive mathematical models.

In the aerospace field, FBGs are under development for structural health monitoring of Carbon Fiber Reinforced Plastics (CFRPs). Conventionally NDT techniques, such as ultrasound C-scan are used, however this requires a long inspection time. Therefore, the development of an NDT technique which can be used for a quick first damage screening is anticipated in the industrial field. One of the candidates for this screening is the analysis of the natural frequency change. In 1975, Adams et al [13], found that the natural frequencies of CFRPs are changed by the existence of damages, causing a reduction in dynamic stiffness. They conducted a modal analysis of the structure using piezoelectric films as both the sensor and actuator. In 2003, Inada et al. [14] identified the location and size of delaminations in CFRP cantilever beams by using both the resonant and anti-resonant frequency. They used a laser displacement meter for the vibration measurement. In the same year, Yam et al. [15] classified the damage location and they identified its severity in composite structures using the vibration responses. They used a piezoelectric patch actuator and sensors for the experiment. In 2002, Takeda et al. [12] used small-diameter FBG sensors for damage detection of CFRPs. They measured the reflection spectra from the FBG sensor for samples with various delamination lengths. They also calculated the reflection spectra numerically. In 2010, Propst et al. [16], demonstrated full-spectral interrogation at $534 \mathrm{~Hz}$ of the signal from a FBG sensor embedded in a composite laminate. They observed peak splitting, wide spectral broadening and a strong single reflection peak due to impact.

In this study, a multi-functional FBG sensor interrogation system which can measure average surface strain, strain distribution and vibration from the reflection spectra has been developed. In order to achieve temperature stabilization, two additional FBG sensors for reference were included. Both the vibration and the average surface strains are measured by peak wavelength shifts of the reflection spectra. Additionally, the distribution of strain is evaluated from the change in width of the reflection spectra.

\section{Theory}

A fibre Bragg grating (FBG) is a periodic modulation of the refractive index within the core of an optical fibre, which couples a forward propagating core mode to a backward propagating core mode at the Bragg wavelength, given by:

$\lambda_{B}=2 n_{e f f} \Lambda$

where $\lambda_{B}, n_{\text {eff }}$ and $\Lambda$ are the reflected Bragg wavelength, the effective refractive index and the grating period, respectively. The refractive index modulation is created when the core of the fibre is exposed to spatially modulated UV irradiation, generally achieved by interfering two UV laser beams [17] or a phase mask [1]. Subsequently, if a spectrally broadband source of light is coupled into the fibre, the FBG reflects a narrow spectral component, typically $0.2 \mathrm{~nm}$ in width, which will also be missing from the transmission spectrum of the fibre. A change in the effective refractive index and/or the grating period will cause a shift in the reflected Bragg wavelength. This forms the principle of measurement of an FBG sensor. The spectral encoding of the measurand by an FBG also allows an array of sensors to be wavelengthdivision-multiplexed, allowing measurements from multiple FBG sensors, with different reflection wavelengths, in one, or more, optical fibres. This is useful for measuring multiple parameters, e.g. strain and temperature, or for measuring at multiple locations. 
When the temperature is constant during the measurement, the relationship between the shift of Bragg wavelength, $\lambda_{\mathrm{B}}-\lambda_{\mathrm{B}_{-} 0}\left(=\Delta \lambda_{\mathrm{B}}\right)$ and the strain, $\varepsilon$ can be expressed in terms of the photoelastic constant, $\mathrm{P}_{\mathrm{e}}$ as [3]:

$\varepsilon=\frac{\lambda_{B}-\lambda_{B_{-} 0}}{\left(1-P_{e}\right) \lambda_{B_{-} 0}}$

Here $\lambda_{\mathrm{B}_{-} 0}$ is the FBG Bragg wavelength with strain and $\lambda_{\mathrm{B}}$ is without strain. As $\mathrm{P}_{\mathrm{e}}$ and $\lambda_{\mathrm{B}_{-} 0}$ are constant during the measurement, $\lambda_{\mathrm{B}}$ has a linear relationship with the strain, $\varepsilon$ which can be expressed as:

$\varepsilon=\alpha \lambda_{B}+\beta$

Temperature-Independent Average Strain Measurement

As mentioned in the Introduction, an additional factor to consider during strain measurement is the temperature sensitivity of the FBG sensor. The temperature effect needs to be compensated for accurate strain measurement. Figure 1 (a) shows the experimental setup used for the experiments. A detailed specification of the setup will be described latter, however it is important to note that three FBGs, connected in series, are used to measure the average strain and vibration measurements. One FBG (shown in $\mathrm{FBG}_{\mathrm{sen}}$ in the figure) was attached to the surface of the specimen and the other two FBGs (referred as $\mathrm{FBG}_{\text {ref1 }}$ and $\mathrm{FBG}_{\text {ref2 }}$ ) are set in the same temperature condition, but not adhered to the specimen. In this system, reflection spectra from the three FBGs are passed through a Fabry-Pérot filter. The distance between the two mirrors in the device is changed with time, and depending on this distance, a narrowband wavelength component passes through the device. By using this tunable filter and an $\mathrm{A} / \mathrm{D}$ converter, three reflection spectrums can be measured over time, as shown in Fig. 1 (b). When the distance between two mirrors is assumed to change linearly with time, the relationship between the peak wavelength, $\lambda_{\mathrm{B}}$ of the reflection spectra and the timing of the peak, $t$ can be described by using two constants of $\alpha$ and $\beta$ as shown in equations (4-6). Subscripts of sen, ref1 and ref2 describe the wavelength or peak arrival time for FBGs used as sensor, reference 1 or reference 2, respectively. A subscript of ${ }_{0}$ describes that the Bragg wavelength is detected without any strain loading except that caused by residual stress due to adhering the FBG to the specimen. Although the Bragg wavelength of $\mathrm{FBG}_{\mathrm{sen}}$ is unknown in the first place, since the residual stress on the $\mathrm{FBG}_{\text {sen }}$ is unknown, it is possible to estimate $\lambda_{\text {Bsen_0 }}$ as well as $\alpha$ and $\beta$ by solving the following equations. This is achieved by substituting known $\lambda_{\text {Bref } 1 \_0}$ and $\lambda_{\text {Bref } 2 \_0}$ values and measured $t_{\text {sen_0 }}$, $t_{\text {ref } 1 \_0}$ and $t_{\text {ref } 2 \_0}$ values.

$\lambda_{\text {Bsen_0 }}=\alpha t_{\text {sen_ } 0}+\beta$

$\lambda_{\text {Bref } 1 \_0}=\alpha t_{\text {ref } 1 \_0}+\beta$

$\lambda_{\text {Bref2_0 }}=\alpha t_{\text {ref } 2 \_0}+\beta$

As $\alpha$ and $\beta$ are not changed during the experiments, the Bragg wavelength with the strain loading conditions, $\lambda_{\text {Bsen_1 }}, \lambda_{\text {Bsen 1_1 }}, \lambda_{\text {Bsen 2_1 (subscript of _1 indicates the }}$ Bragg wavelength during the experiments) can be estimated by using $t_{\text {sen } 11}, t_{\text {ref } 1 \_1}$ and $t_{\text {ref } 21}$ and equations (4-6). The shift in the Bragg wavelength $\Delta \lambda_{B}$ is expressed in equation (7) by the change of strain $\Delta \varepsilon$ and the temperature $\Delta T$ [5]. In reality, the Bragg wavelengths are affected by the temperature drift and $\Delta \lambda_{B}$ can be divided into shifts in Bragg wavelength due to strain $\Delta \lambda_{B_{-} \varepsilon}$ and due to temperature $\Delta \lambda_{B_{-} \text {T. }} K_{\varepsilon}$ and $K_{T}$ are proportional constants for the shift in strain and temperature. When the factor $K_{T}$ is common in three FBGs, the shifts in the Bragg wavelength $\Delta \lambda_{B_{-} T}$ of these three FBGs due to the temperature change become same [see Fig. 1 (b)].

$\Delta \lambda_{B}=K_{\varepsilon} \Delta \varepsilon+K_{T} \Delta T$

$\Delta \lambda_{B}=\Delta \lambda_{B_{-} \varepsilon}+\Delta \lambda_{B_{-} T}$

As a result, the Bragg wavelength, without the effect of temperature, can be measured using following equation.

$\lambda_{\text {Bsen }}=\lambda_{\text {Bref } 1}-\frac{\lambda_{\text {Bref } 1}-\lambda_{\text {Bref } 2}}{t_{r e f 1}-t_{r e f 2}}\left(t_{r e f 1}-t_{s e n}\right)$

As the shift in the Bragg wavelength without the effect of temperature effect has a linear relationship to the strain,
Fig. 1 Experimental setup for multifunctional FBG sensors (a) and the reflection spectra (b). $\mathrm{FBG}_{\text {ref1 }}$ and $\mathrm{FBG}_{\mathrm{ref} 2}$ are used as references for temperature stabilization. $\mathrm{FBG}_{\mathrm{sen}}$ is mounted on the specimen (a)

SLD

(Super Luminescent Diode)

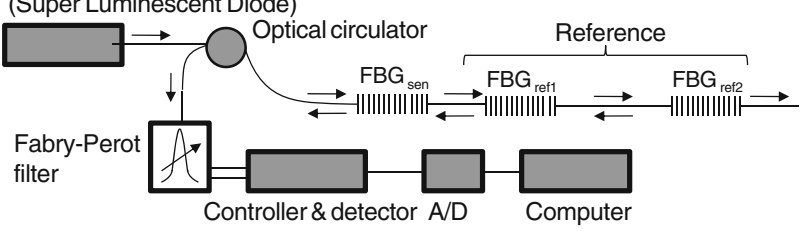

(b)

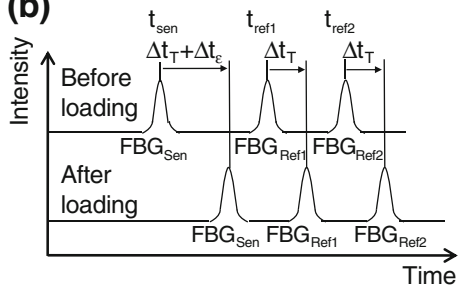


referring to equation (3), the average surface strain $\varepsilon$ experienced by the sensor can be determined.

\section{Strain Distribution Measurement}

Since the grating period in FBGs becomes non-uniform when the object has a strain distribution, the reflection spectra become wider when the strain distribution increases. The Full Width at Half Maximum (FWHM) of the reflection peak becomes larger when the strain distribution increases. Then there is the possibility to realize multiple measurements, a tensile load (by monitoring the shift in peak wavelength of the reflection spectra) and a bending moment (by monitoring the FWHM of the reflection spectra) using one FBG sensor. A general example of this principle is the strain distribution at the surface of a cantilever beam. Figure 2 shows schematically the reason for the FWHM changing with a change in the strain distribution. The Fig. 2 (a) shows the axial surface strain distribution along the sensor and the (c) shows the measured reflection spectra from individual short sections (b) of the grating. As can be seen, individual grating regions reflect different wavelengths. The resulting reflection peaks are superimposed (d), as they cannot be distinguished by the wavelength scanningdetector (Fabry-Pérot filter in Fig. 1). The resulting FWHM value for the reflection spectra is wider when the strain distribution is present. Mathematically the profiles of reflection spectra $(F(\alpha))$ can be approximated by following the Gaussian function. Then a simple model to estimate the FWHM of reflection spectra for the cantilever beam was developed in this study. The coordinate systems and nomenclature used in the following sections are defined in Fig. 3. The sensing part (gauge length) of the FBG is mounted at $x=a$ to $b$ on the beam, which has length $\mathrm{L}$, width $\mathrm{w}$ and thickness $\mathrm{h}$.

$F(\alpha)=\exp \left\{-\frac{4 \ln 2(\alpha-b)^{2}}{c^{2}}\right\}$ here, $\alpha$ is the optical wavelength [nm], $\mathrm{c}$ is the FWHM when no load is applied to the specimen and $b$ is a constant given by the following equation:

$$
\begin{aligned}
b & =\Delta \lambda_{B}+\lambda_{B}=\left(1-P_{e}\right) \lambda_{B} \varepsilon(x)+\lambda_{B} \\
& =\left\{\left(1-P_{e}\right) \varepsilon(x)+1\right\} \lambda_{B}
\end{aligned}
$$

The reflection spectra from an FBG sensor at position $\mathrm{x}$ subject to a strain $\varepsilon(\mathrm{x})$ is:

$F(\alpha)=\exp \left[-\frac{4 \ln 2\left[\alpha-\left\{\left(1-P_{e}\right) \varepsilon(x)+1\right\} \lambda_{B}\right]^{2}}{c^{2}}\right]$

When, the FBG sensor is mounted on the cantilever surface, between positions a to $b$, and subject to a strain $\varepsilon(\mathrm{x})$, the relationship between the wavelength $(\alpha)$ and reflection spectra $(\mathrm{F}(\alpha))$ becomes:

$F(\alpha)=\int_{a}^{b} \exp \left[-\frac{4 \ln 2\left[\alpha-\left\{\left(1-P_{e}\right) \varepsilon(x)+1\right\} \lambda_{B}\right]^{2}}{c^{2}}\right] d x$

Assuming the deflection in the y-direction at $x=0$ is $\delta$, $\varepsilon(\mathrm{x})$ can be determined from the properties of the beam materials using:

$\varepsilon(x)=\frac{3 h \delta}{2 L^{3}} x$

These equations can be solved by numerically with a computer using an iterative process. Figure 4 shows the simulated reflection spectra when $L=0.15[\mathrm{~m}], h=0.001$ $[\mathrm{m}], \lambda_{\mathrm{B}}=1535[\mathrm{~nm}], \mathrm{Pe}=0.22, a=0.145[\mathrm{~m}], b=0.150[\mathrm{~m}]$, $c=0.39[\mathrm{~nm}]$ at deflections $(\delta)$ at $x=0$ of $0.00,0.05$ and $0.10[\mathrm{~m}]$.

As predicted, not only the center wavelength but also the width of the reflection spectra changes with increasing values of $\delta$. This result shows the possibility of determining both the average surface strain and the strain distribution (or bending moment) of cantilever beam independently from the grating reflection spectra.
Fig. 2 Demonstrated the reason for the change in the FWHM value for a grating subject to a linear strain distribution (a)

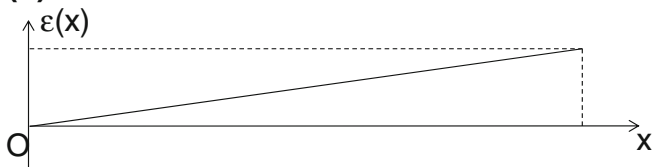

(b)

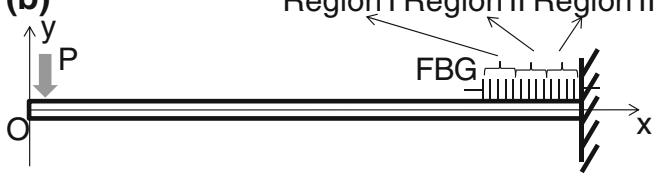

(c)

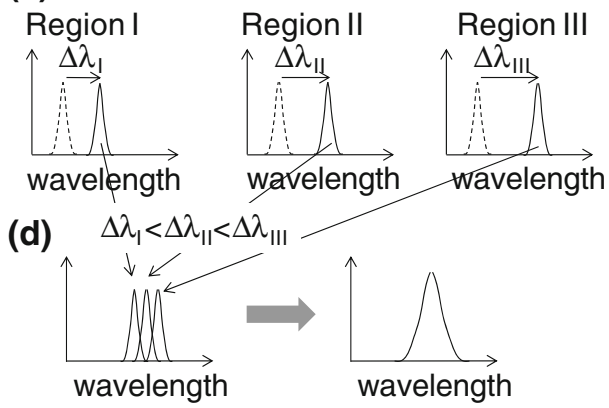


Fig. 3 Definition of the

coordinate system in the model.

The beam has length, L, width,

$\mathrm{w}$, and thickness, h. The FBG

position on the beam is from

$\mathrm{x}=\mathrm{a}$ to $\mathrm{x}=\mathrm{b}$, where $\mathrm{b}-\mathrm{a}$ is the

gauge length of the FBG sensor (a)

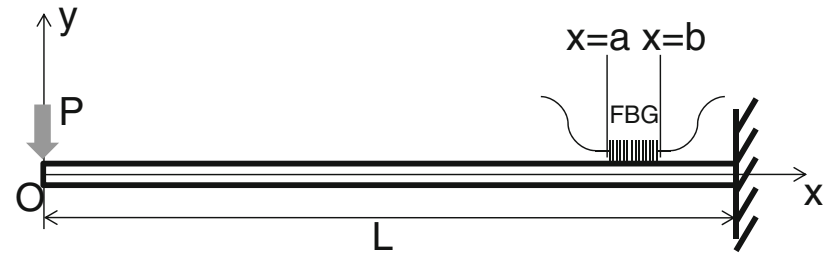

(b)

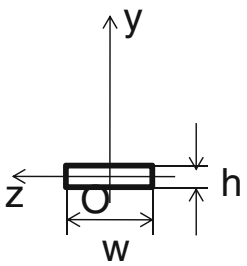

Vibration Measurement

The vibration can be measured by tracking the centre of the FBG sensor reflection peak in time. Figure 5 shows the algorithm for vibration monitoring. Figure 5 (a) shows the movement of mirrors in the Fabry-Pérot filter. The frequency of the mirrors is set to a certain frequency of $\mathrm{F}_{\mathrm{Fab}}[\mathrm{Hz}]$. As the wavelength of light through the filter changes with time, three reflection spectra from $\mathrm{FBG}_{\mathrm{sen}}$, $\mathrm{FBG}_{\mathrm{ref} 1}$ and $\mathrm{FBG}_{\mathrm{ref} 2}$ in Fig. 1 are observed in the forward direction of the mirror movement and other three in the backward direction [see Fig. 5(b)]. Reflection spectra are digitized by an A/D converter at the sampling frequency of $\mathrm{F}_{\text {samp }}[\mathrm{Hz}]$. Then data points of $\mathrm{F}_{\text {samp }} / \mathrm{F}_{\mathrm{Fab}}$ [points] are needed to measure reflection spectra during one cycle of the mirror movement. Although only linear mirror movement is considered in the calculation of wavelength, the reflection spectrum is measured in the forward direction of the mirror movement to take into account the difference in hysteresis between mirror-movement in the forward and backward directions. A dead time is required for the A/D to re-start after the recording the segment of data. As this dead time changes with computer memory usage and CPU occupancy,

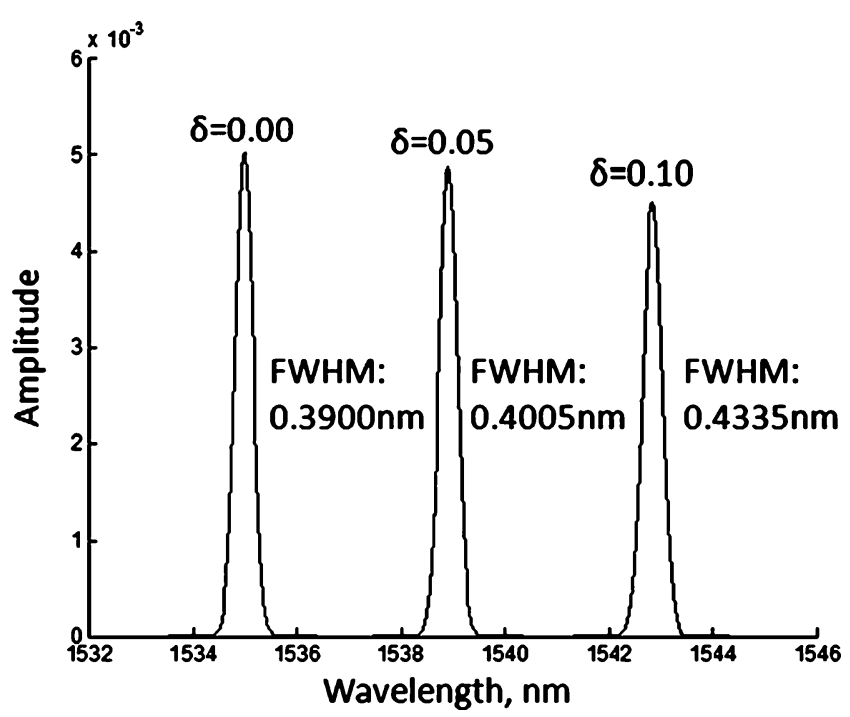

Fig. 4 Simulated reflection spectra for displacements $\delta=0,00,0.05$ and $0.10[\mathrm{~m}]$ at $\mathrm{x}=0$ and has the possibility to miss monitoring next reflection spectra, continuous data measurements are conducted until the A/D reaches its maximum data points (MAX). Then the maximum sampling time becomes MAX/F samp $_{\text {[sec]. When }}$ a long sampling time is required, continuous monitoring techniques have recently been reported in other fields [18]. Vibration can be observed by plotting the wavelength of reflection peak decided by using three spectra measured in the forward direction of the mirror movement [see Fig.(c)]. The sampling frequency of the waveform becomes $\mathrm{F}_{\mathrm{Fab}}$ $[\mathrm{Hz}]$. When high-time resolution is required, frequency of the mirrors $\left[\mathrm{F}_{\mathrm{Fab}}\right]$ should be set in high frequency. On the other hand, the resolution of the amplitude (wavelength) in Fig. 5(c) is decided by the sampling frequency of $A / D$ $\left(\mathrm{F}_{\text {samp }}\right)$, the frequency of $\left(\mathrm{F}_{\mathrm{Fab}}\right)$ and the displacement of the mirror in Fabry-Pérot. One data point of $\mathrm{F}_{\text {samp }} / \mathrm{F}_{\mathrm{Fab}}$ is required to observe one point of data in Fig. 5(c).

\section{Experimental Setup}

Figure 1(a) shows the experimental setup used for the measurement program. A super luminescent diode (Frankfurt Laser Company, $20 \mathrm{~mW}, 1520-1580 \mathrm{~nm}$ wavelength range at FWHM) was used as the light source. Reflection spectra from the FBG sensors were directed via an optical circulator to the scanning Fabry-Pérot interferometer (Micron Optics, Model FFP-TF2) and detector (Micron Optics FFP-C). The signal was digitized by an A/D converter (PICO Technology Model 3224). Digitized data is fed to the computer and is analyzed by software developed using LabVIEW (National instruments). FBG $\mathrm{Fref}_{1}$ and $\mathrm{FBG}_{\text {ref2 }}$ sensors (IXFIBER, wavelengths 1544.81 and $1553.7 \mathrm{~nm}$ ) are used as reference sensors to enable the temperature stabilization. An $\mathrm{FBG}_{\text {sen }}$ sensor (FOS\&S) was attached to the UD-CFRP specimen $\left(400 \mathrm{~mm}^{\mathrm{L}} \times 19.5 \mathrm{~mm}^{\mathrm{W}} \times 1 \mathrm{~mm}^{\mathrm{T}}\right)$ for the cantilever beam test (wavelength: 1,535 $\mathrm{nm}$ ) and the beam for the four point bending rig test (wavelength: $1,540 \mathrm{~nm}$ ), respectively. The position of the FBG sensor on the cantilever beam is at the edge of the specimen where the average and gradient of strain become highest on the beam. On the other hand, the position of the FBG sensor on the four-point bending beam is where the distance from the 
Fig. 5 Sampling of the strain signal from the FBG for vibration monitoring

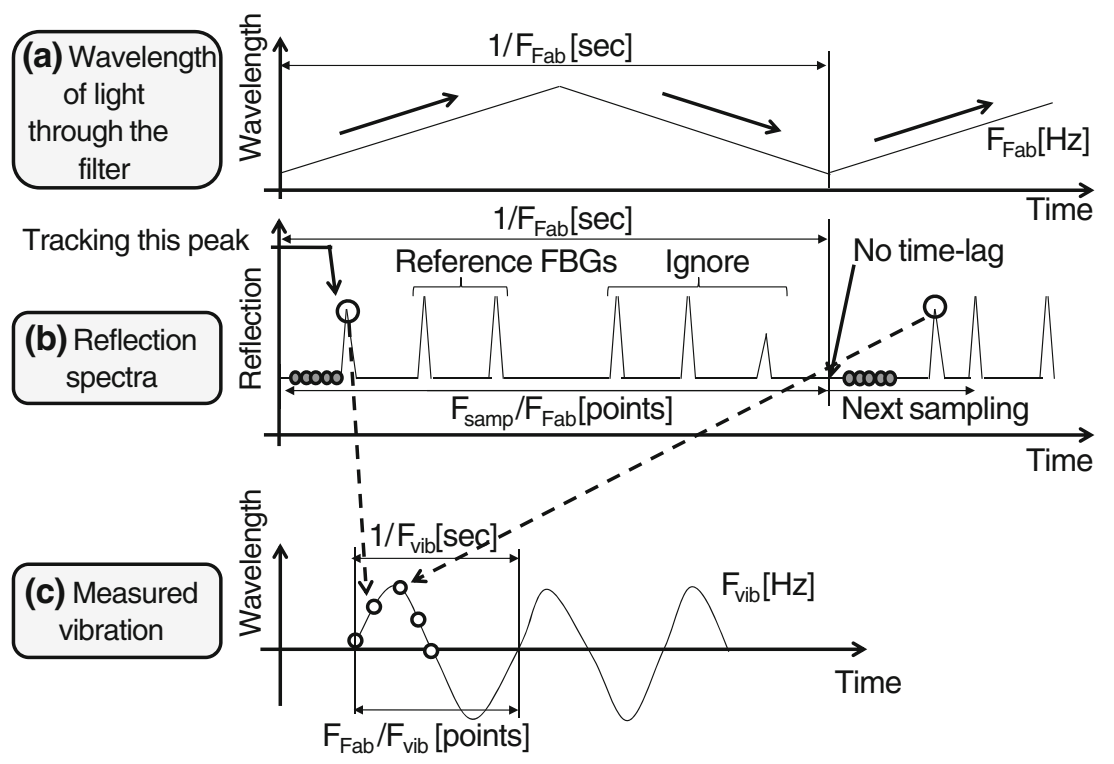

points of application of external forces become farthest as the strain distribution becomes ignorable. Both $\mathrm{FBG}_{\mathrm{sen}}$ sensors are bonded to the top surface of CFRP specimen. The edge of CFRP specimen was firmly clamped. Figure 6 shows the detected reflection spectra by the described system when the $\mathrm{FBG}_{\mathrm{sen}}$ with wavelength 1,535 nm was used. Three large peaks from $\mathrm{FBG}_{\text {sen }}, \mathrm{FBG}_{\mathrm{ref} 1}$ and $\mathrm{FBG}_{\mathrm{ref} 2}$ are observed.

\section{Experimental Results and Discussion}

\section{Average Strain Measurement}

In this study, average surface strains on beams were measured for a cantilever beam test and a four point bending test. The specification of the specimen and the FBG locations are shown in Fig. 7. The specimen for the cantilever beam test is firmly attached to the table at one end. The $\mathrm{FBG}_{\mathrm{sen}}\left(\lambda_{\mathrm{B}}=1535 \mathrm{~nm}\right)$ sensor is bonded to the surface of the specimen at the position $x=211$ to $238 \mathrm{~mm}$ for the cantilever beam test. A resistance strain gauge was attached to the opposite (under) surface at the same location. A uniformly distributed vertical load was applied at the opposite end of the beam (position $x=0$ to $18 \mathrm{~mm}$ ). A second $\mathrm{FBG}_{\mathrm{sen}} \lambda_{\mathrm{B}}=1540 \mathrm{~nm}$ ) sensor was bonded on the centre of the surface of a second specimen for four-point bending test. This specimen also had a resistance strain gauge attached on the opposite (under) surface. The scanning frequency of the Fabry-Pérot interferometer was set to $10 \mathrm{~Hz}$. Data from the detector was digitized at a sampling frequency of $312.5 \mathrm{kHz}$ and blocks of 8,000 sampling points were fed to the computer. The reflection spectra were monitored 30 times for each strain level and the average Bragg wavelengths were determined.

Figure 8 shows monitored reflection spectra from the $\mathrm{FBG}_{\text {sen. }}$. The left figure (a-) shows the results for cantilever beam test and the right (b-) shows those for four-point bending test. Applied strain is shown together with reflection spectra. Wavelengths shown on the horizontal axes are after compensation using the wavelength drift of the reference FBGs. As shown in Fig. 8, peak wavelengths
Fig. 6 Monitored reflection spectra from three FBG sensors

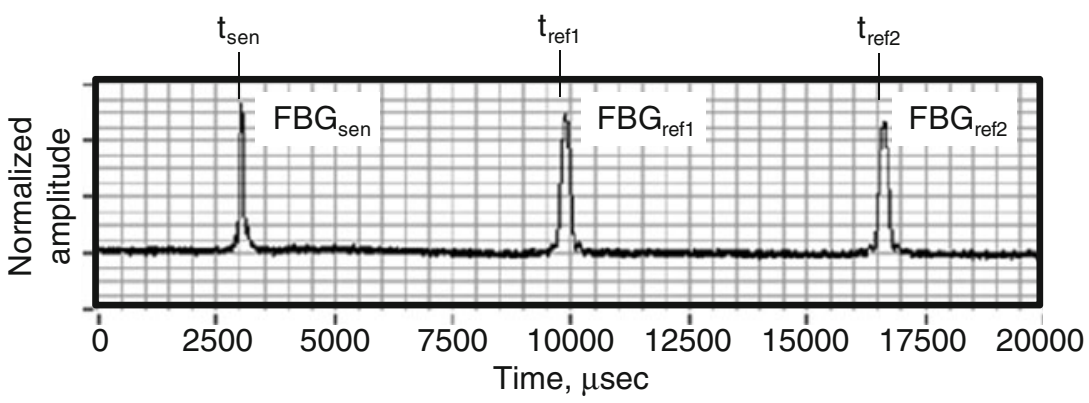


Fig. 7 Specimen and location of FBG sensor for the cantilever beam test (a) and for the fourpoint bending test (b) (a) Cantilever beam test

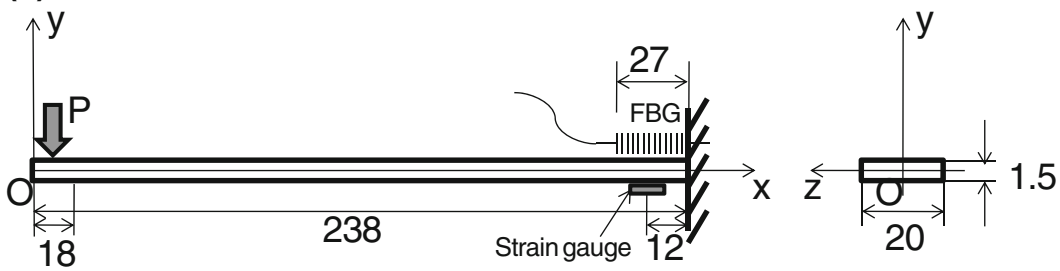

(b) Four-point bending test
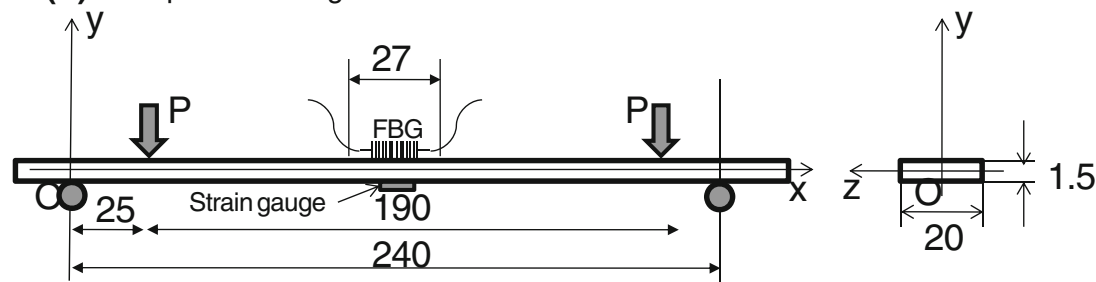

shift with applied strain. Next the data was fitted by $\alpha$ and $\beta$ in equation (3) by the least squares method by using the peak wavelength of the monitored reflection spectra and strain measured by the resistance strain gauge. After that, average strains were estimated using equation (3), by substituting the peak wavelength of monitored reflection spectra. Comparisons of measurement strain by FBG sensor and resistance strain gauge are shown in Fig. 9(a) (for cantilever beam) and (b) (for four-point bending test). Open circles represent surface strain estimated by peak wavelength of monitored reflection spectra and the solid line represents surface strain by resistance gauge for comparison. Open triangle symbols show the difference between them. Average strain is measured within an error of $\pm 20 \mu$ strain. Figure 9 (c) shows wavelength drift by temperature change for the cantilever-beam test. The right axis shows change in strain calculated from wavelength and equation (3) and subtracted by the strain when the temperature is $25^{\circ} \mathrm{C}$. The change in wavelength and strain are $0.042 \mathrm{~nm}$ and $31 \mu$ strain when temperature changes from 25 to $47^{\circ} \mathrm{C}$.

\section{Strain Distribution Measurement}

Strain distributions caused by the cantilever beam test are evaluated from the FWHM of the reflection spectra. The experimental setup and the data acquisition conditions are the same as the previously described experiment. Figure 10 shows the relationship between the FWHM and the strain at the centre of gauge length (Note the sign of the resistance strain gauge reading is reversed as this gauge is in compression). The solid curves shown in the upper part of the figure are calculated results, determined using equation (13). A gauge length of $8 \mathrm{~mm}$ was used in the calculation and the gauge position was $a=0.213 \mathrm{~m}$ and $b=0.221 \mathrm{~m}$. Dots represent experimental results. The error bar shows
Fig. 8 Measured reflection spectra from $\mathrm{FBG}_{\text {sen }}$ at different strain levels. The wavelengths measured are after compensation for temperature changes using $\mathrm{FBG}_{\mathrm{ref} 1}$ and $\mathrm{FBG}_{\mathrm{ref} 2}$ as references
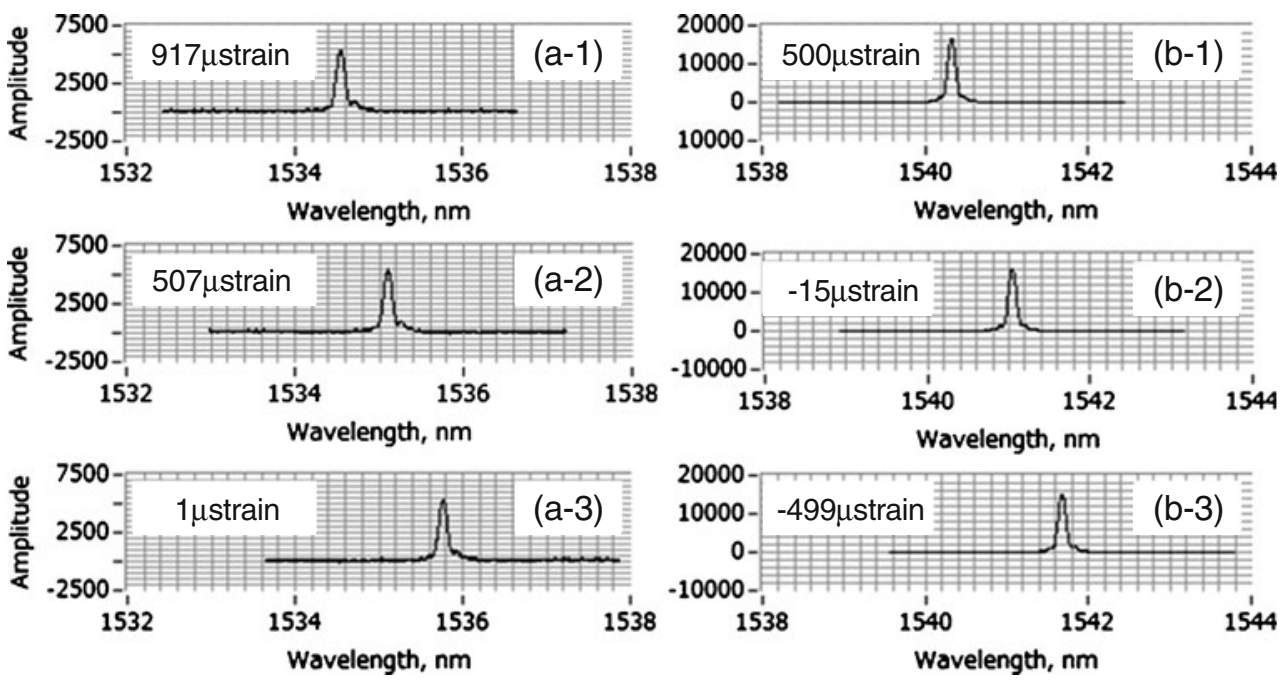


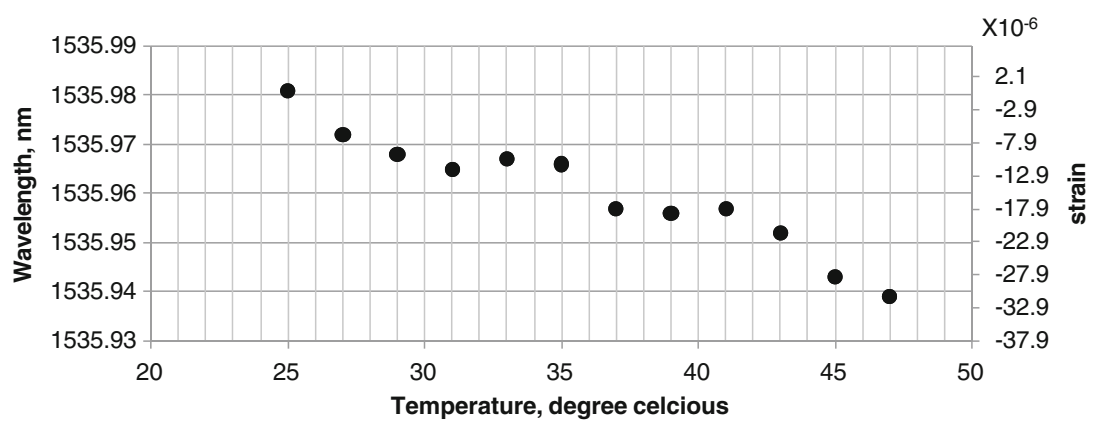

Fig. 9 Estimated surface strain by FBG sensor (open circle), measured surface strain by strain gauge (solid line) and the effect of temperature changing. (a) and (b) show the relationship between the measured surface strain by strain gauge and FBG sensor for the cantilever beam test (a) and four-point bending test (b). The open triangle symbol shows difference between them. (c) shows the effect in strain of drift by temperature change

the standard deviation $(1 \sigma)$ of the experimental data. For the calculation results, represented by the solid lines, the FWHM increased with increasing strain for the cantilever beam test. On the other hand, the FWHM is constant during four-point bending test. This is apparent because the strain changes with $\mathrm{x}$ for the cantilever beam test and is uniform for the four-point bending test. For the experimental data, represented by dots, the change approximates the calculation results, however with a large scatter.

\section{Vibration Measurement}

Vibrations of the cantilever beam were measured using the FBG sensor for different additional masses added to the free end of the cantilever beam. The masses were thin rectangular plates of $200 \times 20 \mathrm{~mm}$ made of aluminum and attached to the plate as it became normal to the plate. During the vibration, these masses vibrated the plate without any movements. In this study, vibration was measured by tracking the peak location of the reflection spectra in time as explained in Fig. 5. The scanning frequency of the Fabry-Pérot interferometer

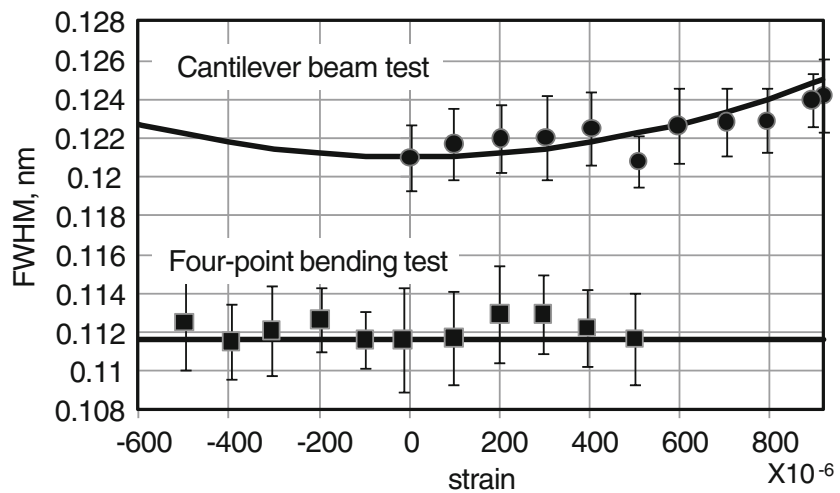

Fig. 10 Calculated FWHM by the proposed equation and measured FWHM at different strain levels
$\left(\mathrm{F}_{\mathrm{Fab}}\right)$ was set to $100 \mathrm{~Hz}$ for this experiment. Distance of the mirrors in the Fabry-Pérot interferometer was set as filtered wavelength became around from 1,532 to 1,557 $[\mathrm{nm}]$. The reflection spectrum was digitized at a sampling frequency $\left(\mathrm{F}_{\mathrm{samp}}\right)$ of $312.5 \mathrm{kHz}$ and the maximum available 256,000 data points [MAX] were fed to the computer. The amplitudes were determined by subtracting the measured wavelength at each timing interval from the average of the local maximum and minimum wavelengths. Figure 11 shows the experimental results. As is shown in the figure, changes of natural frequency by varying the adding mass are observed.

\section{Damage Detection of CFRP Plates}

One application of vibration measurement is to detect damage in CFRP plates. This is demonstrated experimentally by measuring the natural frequency of cantilever beam with a single FBG sensor (the specimen described in previous section was used). An additional mass of $119.7 \mathrm{~g}$ was added to the free end of the cantilever beam. Artificial cracks are introduced and changes of natural frequencies were measured. Figure 12 shows the change of natural frequency for different artificial cracks. The horizontal axis describes cumulative damage. At first the natural frequency of the specimen was measured without damage (shown as "1" in horizontal axis). Artificial surface cracks of $8 \mathrm{~mm}$ length with $0.2 \mathrm{~mm}$ depth were then introduced first at the edge ( $x=238 \mathrm{~mm}$ in Fig. 7(a)) of the specimen (" 2 " of horizontal axis). After that the depth of artificial crack was extended to $0.8 \mathrm{~mm}$ (" 3 "), however, change of natural frequency was small. Then, through cracks of 1, 2, 3, $4 \mathrm{~mm}$ were introduced at the same location ("4"-"7"). After that a $4 \mathrm{~mm}$ through crack was introduced at the centre position of gauge length ( $x=226 \mathrm{~mm}$ in Fig. 7(a), shown as "8" in horizontal axis). Natural frequency decreased with increasing size of artificial cracks. These results show the possibility of using multifunctional FBG sensors for 
Fig. 11 Results of vibration monitoring by changing mass at the end of the cantilever. Open circles represent measured data points

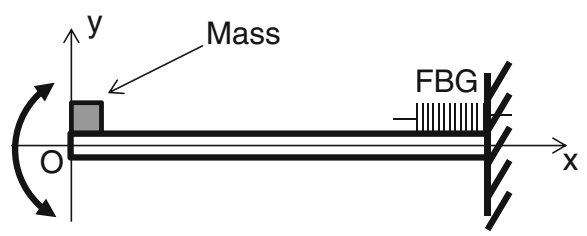

Mass: $56.7 \mathrm{~g}$
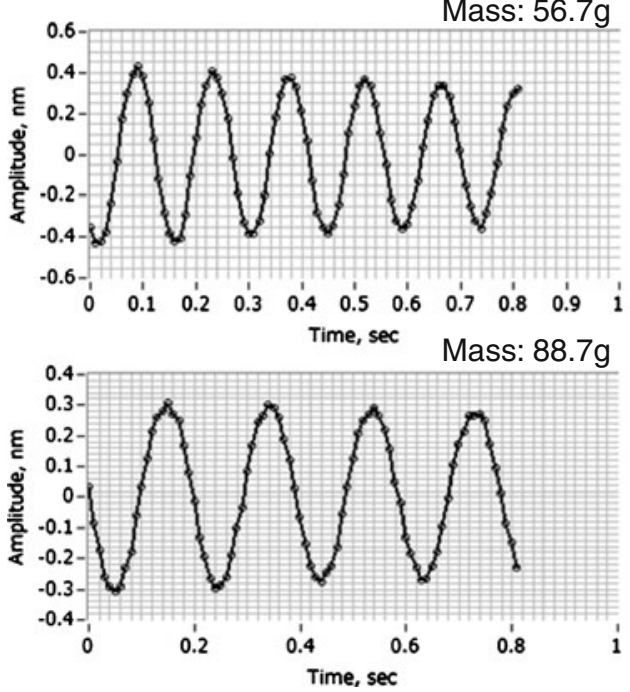

Mass: $119.7 \mathrm{~g}$
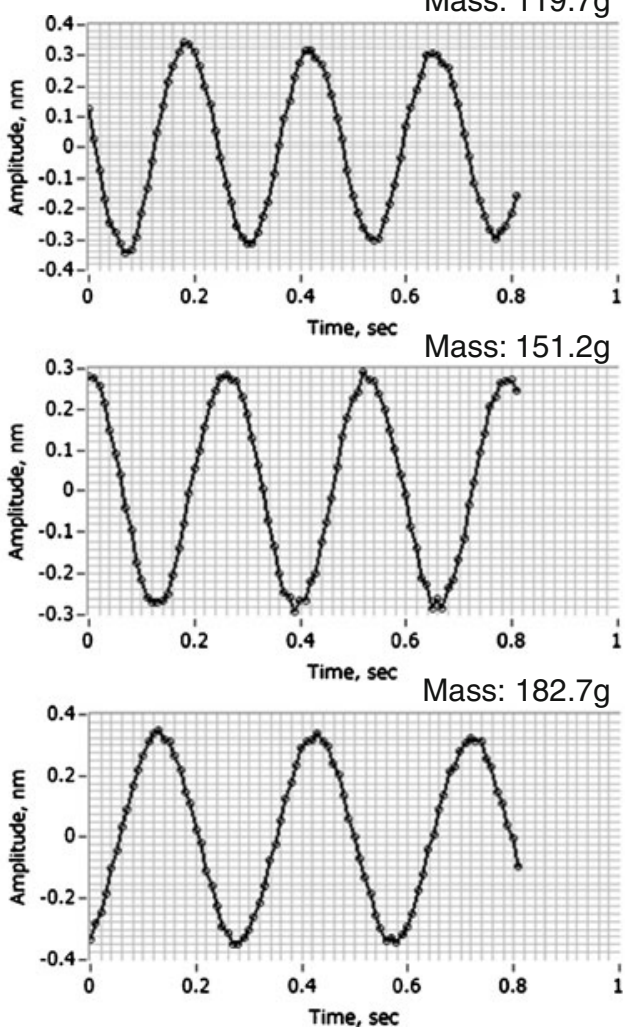

damage monitoring of CFRP structures. The results for 20 repeated tests, conducted to check the reproducibility of the natural frequency measurement, show that the standard deviation of $0.018 \mathrm{~Hz}$ and maximum absolute error of $0.041 \mathrm{~Hz}$. Take into account these error variations, the minimum detectable through crack length will be smaller than $4 \mathrm{~mm}$.

\section{Conclusion}

In this study, average strain, strain distribution and the natural frequency of a cantilever beam made of Carbon

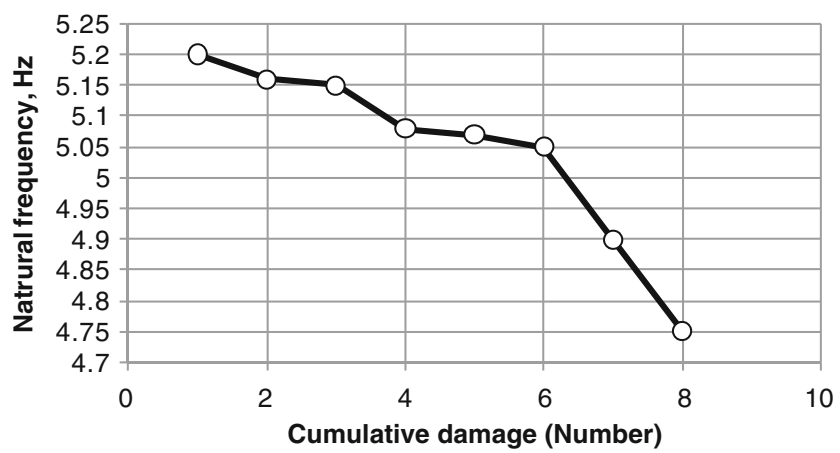

Fig. 12 Natural frequency of specimens with cumulative artificial damage
Fiber Reinforced Plastics (CFRP) were measured using a single FBG sensor mounted on the beam surface. Average strains were estimated from the peak wavelength of the FBG reflection spectrum. By conducting a calibration using surface strain measured by resistance gauge, average strain can be measured accurately by FBG sensors. We confirm that the temperature effects for strain measurement are small using the developed system. Stress distributions are measured by analyzing the FWHM of the reflected light using an analytical model proposed in the paper. The strain distributions measured for the cantilever beam test were compared to those measured for the four point bending test. The trends from the experimental data agree with those from the analytical model. The vibration of a cantilever beam was measured by tracking the location of reflection peak in the spectrum over time. Since the method does not use the amplitude of the spectrum, very stable monitoring can be performed. An application of FBG sensors, damage detection of CFRP plates was demonstrated. A decrease in natural frequency can be detected using FBG sensors.

Acknowledgements This research was conducted during sabbatical visit of Y. Mizutani at Delft University of Technology supported by the Japan Society for the Promotion of Science (Excellent Young Researchers Overseas Visit Program). 
Open Access This article is distributed under the terms of the Creative Commons Attribution Noncommercial License which permits any noncommercial use, distribution, and reproduction in any medium, provided the original author(s) and source are credited.

\section{References}

1. Hill KO, Meltz G (1997) Fiber Bragg grating technology fundamentals and overview. J Lightwave Technol 15-8:1263-1276

2. Rao YJ (1999) Recent progress in applications of in-fibre Bragg grating sensors. Opt Lasers Eng 31:297-324

3. Kersey AD (1996) A review of recent developments in fiber optics sensor technology. Opt Fiber Technol 2:291-317

4. Rao YJ (1998) Fibre Bragg grating sensors: principles and applications. Opt Fiber Sensor Technol 2:355-389

5. Xu MG, Archambault JL, Reekie L, Dakin JP (1994) Discrimination between strain and temperature effects using dualwavelength fibre grating sensors. Electron Lett 30-13:1085-1087

6. Chiang YJ, Wang L, Chen HS, Yang CC, Liu WF (2002) Multipoint temperature-independent fiber-Bragg-grating strainsensing system employing an optical-power-detection scheme. Appl Opt 41-9:1661-1667

7. Takahashi N, Thongnum W, Takahashi S (2003) Fiber-Bragggrating vibration sensor with temperature stability using wavelength-variable incoherent light source. Acoust Sci Technol 23-6:353-355

8. Dias JAS, Leite RL, Ferreira EC (2008) Electronic technique for temperature compensation of fibre Bragg gratings sensors. Int $\mathrm{J}$ Electron Commun 62:72-76
9. Peters K, Studer M, Botsis J, Iocco A, Limberger H, Salathe R (2000) Embedded optical fiber Bragg grating sensor in a nonuniform strain field: measurements and simulation. Exp Mech 19:19-28

10. Prabhugoud M, Peters K (2004) Modified transfer matrix formulation for Bragg grating sensors. J Lightwave Technol 22 10:2302-2309

11. Ling H, Lau K, Cheng L, Chow K (2005) Embedded fibre Bragg grating sensors for non-uniform strain sensing in composite structures. Meas Sci Technol 16:2415-2424

12. Takeda S, Okabe Y, Takeda N (2002) Delamination detection in CFRP laminates with embedded small-diameter fiber Bragg grating sensors. Appl Sci Manuf 33-7:971-980

13. Adams RD, Walton D, Flitcroft JE, Short D (1975) Vibration testing as a non-destructive test tool for composite materials. Composite Reliability, ASTM STP 580:159-175

14. Inada T, Shimamura Y, Todoroki A, Kobayashi H (2003) Development of a two-step delamination identification method using resonant and anti-resonant frequency changes. JSME 69678:231-238

15. Yam LH, Yan YJ, Jiang JS (2003) Vibration-based damage detection for composite structures using wavelet transform and neural network identification. Compos Struct 60:403-412

16. Propst A, Peters K, Zikry MA, Schultz MA, Kunzler MA, Zhu Z, Wirthlin M, Selfridge R (2010) Assessment of damage in composite laminates through dynamic, full-spectral interrogation of fiber Bragg grating sensors. Smart Mater Struct 19:015016

17. Bennion I, Williams JAR, Zhang L, Sugden K, Doran N (1996) UVwritten in-fibre Bragg gratings. Opt Quantum Electron 28:93-135

18. Ito K, Enoki M (2007) Acquisition and analysis of continuous acoustic emission waveform for classification of damage sources in ceramic fiber mat. Mater Trans 48-6:1221-1226 